\title{
Spine-specific skeletal related events and mortality in non-small cell lung cancer patients: a single-institution analysis
}

\author{
*Owoicho Adogwa, MD, MPH, ${ }^{1}$ Daniel R. Rubio, MD,' Jacob M. Buchowski, MD, MS, ${ }^{1}$ \\ Alden D'Souza, BS, ${ }^{1}$ Maksim A. Shlykov, MD, MS, ${ }^{1}$ and Jack W. Jennings, MD, PhD² \\ Departments of ${ }^{1}$ Orthopaedic Surgery and ${ }^{2}$ Radiology, Washington University School of Medicine, St. Louis, Missouri
}

\begin{abstract}
OBJECTIVE The population prevalence of non-small cell lung cancer (NSCLC) continues to increase; however, data are limited regarding the incidence rate of skeletal related events (SREs) (i.e., surgery to the spinal column, radiation to the spinal column, radiofrequency ablation, kyphoplasty/vertebroplasty, spinal cord compression, or pathological vertebral body fractures) and their impact on overall mortality. In this study, the authors sought to estimate the incidence rates of SREs in NSCLC patients and to quantify their impact on overall mortality.
\end{abstract}

METHODS This was a single-institution retrospective study of patients diagnosed with NSCLC between 2002 and 2014. The incidence rates for bone metastasis and subsequent SREs (per 1000 person-years) by time since lung cancer diagnosis were calculated and analyses were stratified separately for each histological type. Incidence rates for mortality at 1,2 , and 3 years from diagnosis stratified by the presence of SREs were also calculated. Kaplan-Meier survival curves were constructed to describe crude survival ratios in patients with spine metastasis and SREs and those with spine metastasis but without SREs. These curves were used to estimate the 1- and 2-year survival rates for each cohort.

RESULTS We identified 320 patients with incident NSCLC (median follow-up 9.5 months). The mean \pm SD age was $60.65 \pm 11.26$ years; $94.48 \%$ of patients were smokers and $60.12 \%$ had a family history of cancer. The majority of firsttime SREs were pathological vertebral body compression fractures (77.00\%), followed by radiation (35\%), surgery (14\%), and spinal cord compression (13.04\%). Mortality rates were highest in NSCLC patients with spine metastasis who had at least 1 SRE. Stratifying by histological subtype, the incidence rate of mortality in patients with SRE was highest in the large cell cohort, 7.42 per 1000 person-years ( $95 \% \mathrm{Cl} 3.09-17.84$ per 1000 person-years); followed by the squamous cell cohort, 2.49 per 1000 person-years ( $95 \% \mathrm{Cl} 1.87-3.32$ per 1000 person-years); and lowest in the adenocarcinoma cohort, 1.68 per 1000 person-years ( $95 \% \mathrm{Cl} 1.46-1.94$ per 1000 person-years). Surgery for decompression of neural structures and stabilization of the spinal column was required in $6 \%$ of patients.

CONCLUSIONS SREs in NSCLC patients with bone metastasis are associated with an increased incidence rate of mortality.

https://thejns.org/doi/abs/10.3171/2020.7.SPINE20829

KEYWORDS lung cancer; non-small cell lung; bone metastases; skeletal related events; spine; pathologic compression fracture; spinal cord compression; oncology

$\mathrm{W}$ ORLDWIDE, lung cancer remains the most common malignancy and the leading cause of cancer-related mortality, with approximately 2.1 million new diagnoses and approximately 1.8 million deaths per year. ${ }^{1}$ Non-small cell lung cancer (NSCLC) represents $85 \%$ of new lung cancer cases and often presents with metastases to distant sites, with a propensity to metastasize to the spine. Spine metastases are reported to occur in $30 \%-40 \%$ of patients with advanced-stage lung cancer, ${ }^{2}$ resulting in significant skeletal morbidity through pathological processes such as spinal cord compression and/or pathological vertebral body fractures and adverse effects from treatments such as surgery or radiation to the spinal column, radiofrequency ablation (RFA), and kyphoplasty/vertebroplasty. Collectively, the cancer-related morbidity associated with bone metastasis is known as skeletal related events (SREs).

SREs continue to pose a significant challenge despite

ABBREVIATIONS NSCLC = non-small cell lung cancer; RFA = radiofrequency ablation; SRE = skeletal related event.

SUBMITTED May 8, 2020. ACCEPTED July 1, 2020.

INCLUDE WHEN CITING Published online November 27, 2020; DOI: 10.3171/2020.7.SPINE20829.

* O.A. and D.R.R. share first authorship. 
improvements in the systemic treatment of NSCLC with the refinement of chemotherapeutic protocols. The presence of SREs increases the morbidity and mortality associated with the underlying lung cancer diagnosis. ${ }^{3}$ Moreover, SREs commonly require additional treatments and hospitalizations that further reduce patient quality of life and increase the total cost of cancer-related care for these patients. ${ }^{4}$ In a retrospective cohort study, Sun and colleagues found that the SREs occurred in the spine in more than $50 \%$ of patients with SREs associated with NSCLC; ${ }^{5}$ thus, spine surgeons are frequently involved in the care of these patients.

Despite the prevalence and impact associated with SREs in the care of patients with NSCLC, there is a paucity of literature currently available on NSCLC spine-specific SREs. The goal of the present study was to estimate the incidence, morbidity, and mortality associated with spine-specific SREs.

\section{Methods \\ Patient Sample}

This was a retrospective single-institution cohort study conducted at a tertiary academic center in the United States (Washington University in St. Louis, MO). There were 320 patients enrolled in the study. Institutional Review Board approval was obtained prior to initiation of the study.

\section{Lung Cancer}

We identified patients with a first primary diagnosis of NSCLC recorded between January 1, 2002, and December 31, 2014. Data on patient demographics, location of tumor, morphology, and stage at diagnosis were collected for all patients. The NSCLC stage was categorized by the presence of distant metastasis at diagnosis. Histological subtypes were classified as follows: adenocarcinoma, squamous cell carcinoma, large cell carcinoma, and other.

\section{Spine Metastasis and SREs}

As part of the comprehensive oncological workup, all patients in this study had CT scans of the chest, abdomen, and pelvis. In patients with spinal metastasis, dedicated CT scans of the cervical, thoracic, and lumbar spine were obtained. These scans were used to identify spinal metastasis and assist in disease staging. In addition, information on the type of SREs and location was obtained for all patients. SREs were defined as follows: surgery to the spinal column, radiation to the spinal column, RFA to the vertebral column, kyphoplasty/vertebroplasty, spinal cord compression, or pathological vertebral body fractures. Only SREs recorded in connection with or after a spine metastasis diagnosis were included. In addition, patients could have $>1$ SRE.

\section{Comorbidities}

Data on smoking status and comorbidities were collected for all patients. The number of self-reported cigarette packs per day smoked and the number of years smoked were used to calculate a smoking pack-year history. Data on the presence of comorbidities such as atrial fibrillation, diabetes mellitus, congestive heart failure, myocardial infarction, deep venous thrombosis, pulmonary embolism, and peripheral vascular disease were collected on all patients.

\section{Neurological Status}

Data on neurological status at the time of diagnosis and subsequent follow-up visits were recorded for each patient. Patients were categorized into one of the following four groups: 1) complete spinal cord injury, 2) incomplete spinal cord injury, 3) neurologically intact, or 4) nerve root compression causing symptomatic radiculopathy.

\section{Mortality}

Mortality data were obtained from the electronic medical record system. Patients were followed from date of lung cancer diagnosis to date of death.

\section{Statistical Analysis}

Incidence rates for spine metastasis and subsequent SREs (per 1000 person-years) by time since lung cancer diagnosis were calculated and analyses were conducted separately for each histological type. We calculated the incidence rates for mortality at 1,2 , and 3 years from diagnosis stratified by the presence of SREs.

Kaplan-Meier survival curves were constructed to describe crude survival ratios in patients with spine metastasis and SREs and those with spine metastasis but without SREs. These curves were used to estimate the 1- and 2 -year survival rates for each cohort. Follow-up began on the date of lung cancer diagnosis, and patients contributed time at risk in each cohort until they became eligible for a different cohort. We also calculated crude mortality rates (deaths per 1000 person-years) according to the presence of SREs stratified by major histological subtype.

Parametric data were expressed as means \pm SDs and compared via Student t-tests. Nonparametric data were expressed as median (IQR) and compared via the MannWhitney U-test, and $95 \%$ confidence intervals were also calculated. All tests were two-sided and considered statistically significant at $p<0.05$. The statistical analysis was performed using JMP (version 12; SAS Institute Inc.).

\section{Results}

Table 1 presents baseline characteristics of the study cohort. In total, 320 patients with a diagnosis of NSCLC and spinal metastasis were included in the study, of whom 45 (14\%) patients were lost to follow-up. The missing data were considered missing completely at random (MCAR) and did not bias the results. The mean \pm SD age was $60.65 \pm 11.26$ years, with a median follow-up of 9.5 months (IQR 4.3-22.0 months); $51.87 \%$ of patients were men, $94.48 \%$ were smokers, and $60.12 \%$ had a family history of cancer. The mean \pm SD number of years smoked was $32.72 \pm 12.73$ years. The most common histological subtype was adenocarcinoma (77.86\%), followed by squamous cell carcinoma (19.29\%) and large cell carcinoma $(2.86 \%)$. 
TABLE 1. Characteristics of NSCLC patients overall and by SREs

\begin{tabular}{|c|c|c|c|}
\hline Variable & $\begin{array}{l}\text { NSCLC Cohort } \\
\qquad(n=320)\end{array}$ & $\begin{array}{l}\text { NSCLC Patients w/ Bone } \\
\text { Metastasis \& SRE }(n=100)\end{array}$ & $\begin{array}{c}\text { NSCLC Patients w/ Bone } \\
\text { Metastasis \& w/o SRE }(n=220)\end{array}$ \\
\hline Male & $166(51.87)$ & $49(49.00)$ & $117(53.18)$ \\
\hline Patient age, yrs, mean \pm SD & $60.65 \pm 11.26$ & $61.29 \pm 10.58$ & $60.36 \pm 11.56$ \\
\hline History of smoking & $240(75.00)$ & $95(95.00)$ & $205(93.92)$ \\
\hline Family history of cancer & $190(59.37)$ & $58(58.00)$ & $132(60.00)$ \\
\hline Family history of NSCLS & $66(20.62)$ & $22(22.00)$ & $44(20.00)$ \\
\hline \multicolumn{4}{|l|}{ Histology } \\
\hline Adenocarcinoma & $218(77.86)$ & $64(74.42)$ & $154(79.38)$ \\
\hline Squamous & $54(19.29)$ & $17(19.77)$ & $37(19.07)$ \\
\hline Large cell & $8(2.86)$ & $5(5.81)$ & $3(1.55)$ \\
\hline \multicolumn{4}{|l|}{ Preexisting comorbidity } \\
\hline Diabetes & $44(13.75)$ & $11(11.00)$ & $33(15.00)$ \\
\hline $\mathrm{CHF}$ & $11(3.44)$ & $0(0.00)$ & $11(5.02)$ \\
\hline MI & $21(6.60)$ & $6(6.00)$ & $15(6.88)$ \\
\hline $\mathrm{PE}$ & $43(13.43)$ & $10(10.00)$ & $33(15.00)$ \\
\hline Afib & $23(7.18)$ & $8(8.00)$ & $15(6.81)$ \\
\hline PVD & $5(1.56)$ & $0(0.00)$ & $5(2.27)$ \\
\hline
\end{tabular}

$\mathrm{Afib}=$ atrial fibrillation; $\mathrm{CHF}=$ congestive heart failure; $\mathrm{MI}=$ myocardial infarction; $\mathrm{PE}=$ pulmonary embolism; $\mathrm{PVD}=$ peripheral vascular disease .

Values are presented as number (\%) of patients unless otherwise indicated.

\section{Clinical Presentation}

At presentation, the majority of patients had more than 4 metastatic lesions to the spine, with the most common regions being the thoracic and lumbar spine (Table 2); $77.00 \%$ of patients had a pathological vertebral body fracture, of which $64 \%$ were assessed to be stable fractures and $36 \%$ unstable compression fractures. The vast majority $(74.68 \%)$ of patients at presentation were neurologically intact (Table 3).

One hundred (31.25\%) patients with NSCLC had at least 1 SRE following the diagnosis of spinal metastasis. The majority of first-time SREs were pathological vertebral body fractures $(77.00 \%)$, followed by radiation $(35 \%)$, surgery (14\%), and spinal cord compression (13.04\%). Patients could have more than 1 SRE and were counted accordingly. SREs most commonly occurred in the thoracic spine (51.33\%) and lumbar spine (26.55\%) (Table 4). The mean time from diagnosis to an SRE was $14.90 \pm 24.75$ months. By histological subtype, rates of SREs were highest in adenocarcinoma patients, followed by squamous cell carcinoma and then large cell carcinoma patients.

In regard to treatment of SREs, $35.0 \%$ of patients received conventional radiation, $36.0 \%$ underwent cement augmentation of the vertebral body ( $29.0 \%$ vertebroplasty, $7.0 \%$ kyphoplasty), and $14.0 \%$ required surgery (Table 4 ).

\section{Mortality Rates}

Mortality rates were lower in lung cancer patients with spine metastasis and no SRE and generally highest in lung cancer patients with spine metastasis who had at least 1 SRE. Stratifying by histological subtype, the incidence rate of mortality in patients with SRE was highest in the large cell cohort (7.42 per 1000 person-years; 95\% CI 3.09-17.84 per 1000 person-years), followed by the squamous cell cohort (2.49 per 1000 person-years; $95 \%$ CI 1.87-3.32 per 1000 person-years) and lowest in the adenocarcinoma cohort (1.68 per 1000 person-years; 95\% CI 1.46-1.94 per 1000 person-years) (Table 5). The observed association between the histological subtype and the incidence rate of mortality appeared consistent at 1,2, and 3 years after lung cancer diagnosis (Table 6).

\section{Survival}

Kaplan-Meier survival estimates were constructed for two dynamic lung cancer cohorts: spinal metastasis with SRE and spinal metastasis without SRE. The 1- and 2-year survival estimates were similar between both cohorts $(p=0.43)$ (Fig. 1). Similarly, Kaplan-Meier survival estimates demonstrating crude survival estimates in lung cancer patients by major histological subtypes were constructed for three dynamic and distinct histological groups: adenocarcinoma, squamous cell carcinoma, and large cell carcinoma. The 1- and 2-year survival estimates were significantly different between the groups, with the shortest survival estimate occurring in patients with large cell carcinoma (Fig. 2).

\section{Surgery}

Of the 320 patients enrolled in the study, 5.90\% required primary surgery. Of the patients requiring surgery, $73.68 \%$ were neurologically intact at the time of surgery, $21.05 \%$ had an incomplete neurological injury, and $5.26 \%$ had a complete neurological injury. The most common histological subtype in patients who required surgery was adenocarcinoma (52.6\%), followed by squamous cell carcinoma $(36.8 \%)$ and large cell carcinoma $(10.5 \%)$. The median number of levels fused was 5 (IQR 4-6 levels). The 
TABLE 2. Summary of clinical and radiographic variables at presentation in patients with NSCLC with or without SREs

\begin{tabular}{|c|c|c|}
\hline Variable & NSCLC Patients w/ Bone Metastasis \& SRE $(n=100)$ & NSCLC Patients w/ Bone Metastasis \& w/o SRE $(n=220)$ \\
\hline Smoking, yrs, mean \pm SD & $35.23 \pm 12.07$ & $31.39 \pm 12.90$ \\
\hline \multicolumn{3}{|c|}{ Neurological status on presentation } \\
\hline Intact & 58.76 & 81.86 \\
\hline Incomplete SCI & 14.43 & 3.73 \\
\hline Complete SCl & 3.09 & 0.47 \\
\hline Radiculopathy & 23.71 & 13.95 \\
\hline \multicolumn{3}{|l|}{ No. of spine lesions } \\
\hline$>0$ & 24.00 & 35.45 \\
\hline$>2$ & 6.00 & 10.45 \\
\hline$>4$ & 70.00 & 52.72 \\
\hline \multicolumn{3}{|l|}{ Spine lesion location } \\
\hline Cervical & 1.01 & 3.21 \\
\hline Thoracic & 14.14 & 28.44 \\
\hline Lumbar & 7.07 & 10.55 \\
\hline Cervicothoracic & 3.03 & 3.66 \\
\hline Thoracolumbar & 42.42 & 43.11 \\
\hline Cervical/thoracic/lumbar & 32.23 & 11.00 \\
\hline \multicolumn{3}{|l|}{ Spine lesions } \\
\hline Asymptomatic & 54.83 & 66.50 \\
\hline Uncomplicated pain & 45.16 & 33.49 \\
\hline \multicolumn{3}{|l|}{ Vertebral body fracture } \\
\hline Stable & 63.51 & 58.33 \\
\hline Unstable & 36.48 & 41.66 \\
\hline
\end{tabular}

Values are presented as percentages of patients unless otherwise indicated.

mean \pm SD length of surgery was $248 \pm 95$ minutes, and the mean \pm SD intraoperative estimated blood loss was $606 \pm 682 \mathrm{~mL}$. The median length of in-hospital stay was 5.5 days (IQR 4-7 days). Postoperatively, 1 patient had a pulmonary embolism, 1 had a wound infection requiring reoperation, and 1 patient had a wound dehiscence. Five (26.31\%) patients were readmitted within 30 days of surgery and $6(31.57 \%)$ within 90 days of surgery.

TABLE 3. Type and location of SRES

\begin{tabular}{cc}
\hline \multicolumn{1}{c}{ SREs $(n=100)$} & No. $(\%)$ of Patients \\
\hline Type & \\
\hline PVBF & $77(77.00)$ \\
\hline SCC & $8(8.00)$ \\
\hline PVBF causing SCC & $14(14.00)$ \\
\hline Nerve root compression & $1(1.00)$ \\
\hline Level & $3(2.65)$ \\
\hline Cervical & $58(51.33)$ \\
\hline Thoracic & $30(26.55)$ \\
\hline Lumbar & $4(3.54)$ \\
\hline Cervicothoracic & $15(13.27)$ \\
\hline Thoracolumbar & $3(2.65)$ \\
\hline Cervical/thoracic/lumbar
\end{tabular}

PVBF = pathological vertebral body fracture; SCC = spinal cord compression .

\section{Discussion}

Lung cancer remains the most common malignancy and leading cause of cancer-related mortality worldwide, with the vast majority of new lung cancer cases of the NSCLC subtype. ${ }^{1}$ Spine metastases occur in $30 \%-40 \%$ of patients with advanced-stage lung cancer, and this predilection for the spine places patients at risk for the skeletal morbidities known collectively as SREs, including surgery to the spinal column, radiation to the spinal column, RFA, kyphoplasty/vertebroplasty, spinal cord compression, and pathological vertebral body fractures.,5 These SREs not only increase morbidity and mortality, but also reduce quality of life and increase the total cost of cancerrelated care. ${ }^{3,4}$ Despite the prevalence and impact of spinerelated SREs, little information has been published on the incidence, morbidity, mortality, and management of spinespecific SREs. The goal of the present study is to address this paucity of literature regarding spine-specific SREs.

The present study was a single-institution retrospective cohort study at a large academic cancer referral center. A total of 320 consecutive patients with a known diagnosis of NSCLC, known metastasis to the spine, and a mean age of 60.6 years and a median follow-up of 9.5 months were enrolled between 2002 and 2014. Approximately $95 \%$ of patients had a history of tobacco smoking, with average pack-years smoked by patients with and without SRE at presentation of 35.2 pack-years and 31.4 packyears, respectively (Table 1). At presentation, the majority 
TABLE 4. Treatments administered to patients with NSCLC with spinal metastasis

\begin{tabular}{lcc}
\hline \multicolumn{1}{c}{ Variable } & $\begin{array}{c}\text { NSCLC Patients w/ Bone } \\
\text { Metastasis \& SRE }(\mathrm{n}=100)\end{array}$ & $\begin{array}{c}\text { NSCLC Patients w/ Bone } \\
\text { Metastasis \& w/o SRE }(\mathrm{n}=220)\end{array}$ \\
\hline Systemic chemotherapy & $75.00(65.57-82.53)$ & $79.16(73.21-84.08)$ \\
\hline Anterior vertebral body involvement & $84.00(75.42-89.97)$ & $0.40(0.00-3.10)$ \\
\hline Posterior column involvement & $48.00(38.37-57.78)$ & $0.40(0.00-3.10)$ \\
\hline Spinal metastasis & & \\
\hline RFA & $9.00(4.73-16.43)$ & $0.90(0.00-3.50)$ \\
\hline Cryoablation & $5.00(2.00-11.48)$ & $0.90(0.20-3.57)$ \\
\hline Vertebroplasty & $29.00(20.93-38.65)$ & $0.09(0.06-4.75)$ \\
\hline Kyphoplasty & $7.00(3.36-13.99)$ & $0.04(0.06-3.17)$ \\
\hline SRS spine & $7.00(3.36-13.99)$ & $2.72(1.20-5.95)$ \\
\hline Conventional RT to spine & $35.00(26.27-44.85)$ & $20(15.21-25.83)$ \\
\hline Surgery & $14.00(8.4-22.29)$ & $2.28(0.09-5.38)$ \\
\hline
\end{tabular}

$\mathrm{RT}=$ radiation therapy; SRS = stereotactic radiosurgery.

Values are presented as mean $(95 \% \mathrm{Cl})$. The mean \pm SD time to SRE was $14.90 \pm 24.75$ months.

of patients had at least 4 sites of osseous metastases to the spine in both cohorts, with and without SREs (Table 2). In the cohort with SREs, $8.33 \%$ of patients presented with spinal cord injury (7.05\% incomplete and $1.28 \%$ complete spinal cord injury), $45.2 \%$ of patients presented with uncomplicated neck and/or back pain, and $36.4 \%$ of patients presented with an unstable pathological vertebral body fracture (Table 2). There was no significant difference in the 1- and 2-year mortality rates between patients with NSCLC with known metastasis to the spine with and those without SRE.

The literature on the incidence of spine metastases and SREs and their impact on survival in patients with NSCLC is limited and broad in focus. The majority of the prior research utilized large national databases. In one of the largest retrospective cohort studies, Sathiakumar and colleagues found that approximately $20 \%$ of patients with NSCLC developed osseous metastases, and approximately $50 \%$ of patients with known bone metastases experienced SREs. ${ }^{6}$ This prevalence of SREs in patients with known bone metastases has been echoed in multiple similar large database studies. ${ }^{3,4,7}$ In most of these large database studies, the most common SRE is radiation to the bone, which occurred in $67 \%$ to $79 \%$ of NSCLC patients who experienced SRE. 3,4,6,7 The prevalence of surgery in these large database studies ranges from $1 \%$ to $14 \%$ of patients. $3,4,6,7$ Across these large database studies, comparisons of SRE incidence and survivability after SRE are limited given the variety of definitions utilized. Cetin and colleagues found that the SRE incidence rate after diagnosis of spine metastases was 3064 per 1000 person-years, and only $5.1 \%$ of these patients survived 1 year. ${ }^{3}$ In contrast, Pockett and colleagues found that the SRE incidence rate following diagnosis of NSCLC was 260 per 1000 person-years. ${ }^{8}$

There are few published single-institution retrospective cohort studies that explore the incidence and impact of SREs in NSCLC patients. Tsuya and colleagues discovered that approximately $30 \%$ of patients will develop osseous metastases within 3 years of the diagnosis of NSCLC. ${ }^{9}$ In their study, these authors determined that the median time to SRE was 3.0 months and the median survival time after the first SRE was 3.0 months. Sekine and colleagues found that approximately $20 \%$ of patients will develop SREs within 6 years of the diagnosis of NSCLC..$^{10}$ In another study, Sun and colleagues revealed that approximately $60 \%$ of patients experienced an SRE within 3 years after the diagnosis of osseous metastases. ${ }^{5}$ In their study, they found that the prevalence rates of SREs in the spine, pelvis, extremities, and chest wall were $55.2 \%$, $24.5 \%, 17.1 \%$, and $5.3 \%$, respectively. In all these singleinstitution retrospective cohort studies, the majority of SREs were radiation to the bone, with the incidence ranging from $34.3 \%$ to $91.0 \% .^{5,9,10}$

The impact of SREs on overall survival remains unclear. Da Silva et al., ${ }^{11}$ in a retrospective series of 95 patients with a median follow-up of 4.4 months, found that

TABLE 5. Incidence rates of mortality by SRES

\begin{tabular}{lccc}
\hline \multicolumn{1}{c}{ Variable } & Person-Yrs & Incidence Rate & $95 \% \mathrm{Cl}$ \\
\hline SRE & 49,514 & 1.61 & $1.29-2.01$ \\
\hline No SRE & 131,750 & 1.38 & $1.20-1.60$ \\
\hline Mortality/1000 person-yrs by histology & & & \\
\hline Adenocarcinoma & 112,770 & 1.68 & $1.46-1.94$ \\
\hline Squamous cell & 18,833 & 2.49 & $1.87-3.32$ \\
\hline Large cell & 673 & 7.42 & $3.09-17.84$ \\
\hline
\end{tabular}


TABLE 6. Incidence rate of mortality by SREs and major histologic subtype by time since lung cancer diagnosis

\begin{tabular}{lccc}
\hline \multirow{2}{*}{ Variable } & \multicolumn{3}{c}{ Yr After Diagnosis } \\
\cline { 2 - 4 } & \multicolumn{1}{c}{ 1st } & 2nd & 3rd \\
\hline Mortality/1000 person-yrs by SREs & & & \\
\hline SRE & $2.14(1.58-2.89)$ & $1.56(0.96-2.56)$ & $1.80(0.97-3.36)$ \\
\hline No SRE & $2.37(1.96-2.87)$ & $1.85(1.33-2.57)$ & $1.16(0.69-1.97)$ \\
\hline Mortality/1000 person-yrs by histology & & & \\
\hline Adenocarcinoma & $2.14(1.77-2.60)$ & $1.47(1.06-2.05)$ & $1.47(0.96-2.25)$ \\
\hline Squamous cell & $2.48(1.73-3.55)$ & $3.29(1.82-5.95)$ & $1.85(0.59-5.74)$ \\
\hline Large cell & $6.34(2.38-16.91)$ & $23.25(3.27-165.09)$ & - \\
\hline
\end{tabular}

Values are presented as mean $(95 \% \mathrm{Cl})$.

the overall survival time was lower in patients who experienced SREs. In contrast, Tominaga et al., ${ }^{12}$ in a study of 85 patients, of whom 47 presented with SREs, found no statistically significant difference in overall survival between the patients who experienced SREs and those who did not. In our study, the incidence rate of mortality was higher in patients who experienced an SRE; however, the overall survival time was not significantly different between the SRE and non-SRE cohorts $(\mathrm{p}=0.43)$.

To the authors' knowledge, this study is the first that explores the incidence and impact of spine-specific SREs. Similar to SREs reported in prior studies, the majority of SREs were radiation to the bone, which occurred in $35.0 \%$ of patients; however, the prevalence in this study is more conservative than the rates reported in the existing literature. This difference suggests that radiation to the bone may be more commonly administered to bones outside of the spinal column, such as the pelvis and extremities. Additionally, the prevalence of surgery to the spine in the present study was $14 \%$, which is similar to the surgery prevalence rates reported in the existing literature, which range from $1 \%$ to $14 \% .4,6,7,9,10$ Moreover, the prevalence of spinal cord compression in this study was $20.8 \%$, which is also similar to the prevalence rates in other published reports, ranging from $3 \%$ to $21 \% .^{4-7,9,10}$ The similarities in SRE prevalence in this study suggest that the current cohort can potentially be used for future study of SRE impact in this unique population.

The main strength of this study is the fact that it is a single-institution study. This allows for patient-specific risk factors and outcomes to be studied that cannot be studied in large database studies. These large database studies lack the granular patient-specific data needed to make firm conclusions because they utilize ICD and CPT codes to assess risk factors and outcomes, and therefore the results may be subject to coding inaccuracies. Moreover, review of medical records allows for spine-specific risk factors and outcomes to be studied. The main limi-

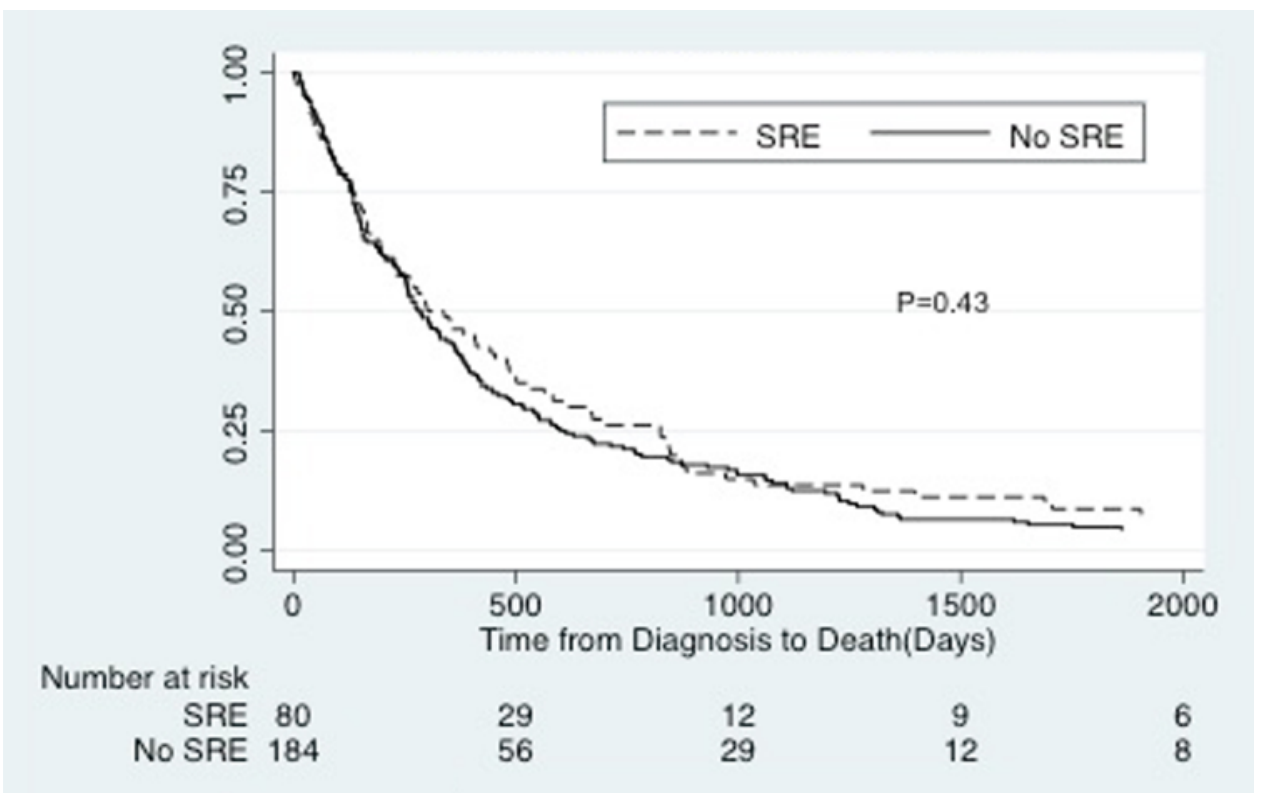

FIG. 1. Kaplan-Meier survival curves demonstrating crude survival estimates in NSCLC patients with bone metastasis with and without SREs. Figure is available in color online only. 


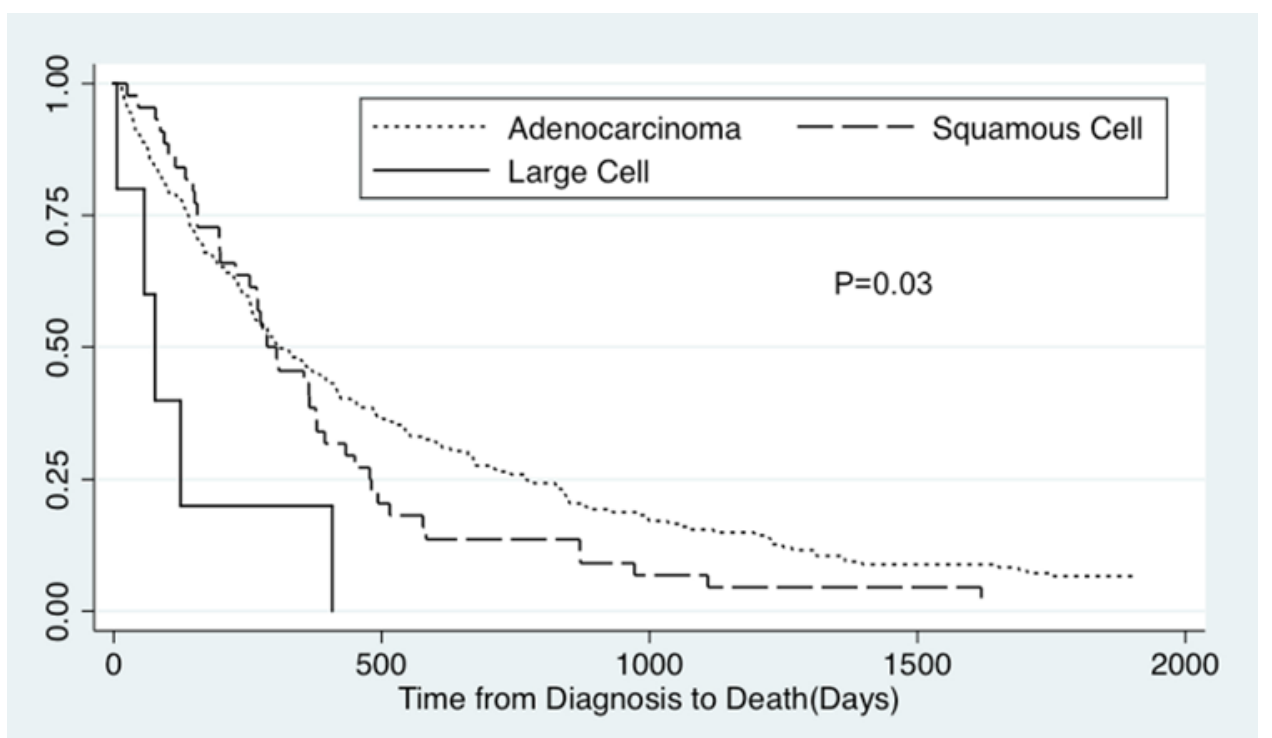

FIG. 2. Kaplan-Meier survival curves demonstrating crude survival estimates in NSCLC patients by major histological subtypes. Figure is available in color online only.

tation of the present study is that it is retrospective, and thus subject to selection bias and confounding that could limit the application of the results to a broad population of NSCLC patients. However, the creation of a prospective study exploring SREs in NSCLC patients may not be possible given the overall low incidence of NSCLC SREs in the general population.

There are some broad trends that we can glean from this single-institution series over the time period of the study. The mean durations of time from diagnosis to development of an SRE were 28 months (2002-2005), 11 months (2006-2010), and 13 months (2011-2012), respectively. These three time points are considered separately for several reasons. Pathological compression fractures as well as fractures causing spinal cord compression were the most common SREs across all three time points; however, stereotactic radiosurgery was used at a higher frequency from 2006 onward compared with prior to 2006, although conventional radiation therapy was still most commonly used across all three time points. Cement augmentation was used at a higher frequency after 2010 (13\%) compared with 2002-2010 (9\%). RFA and cryoablation were used at a higher frequency in the years after 2012 than during the years prior. Molecular markers such as EGFR, ALK, BRAT, POS1, and PDL1 were captured at a higher frequency after 2006, although it is not clear how this information has been used to guide treatment. Similarly, primary surgery was performed for decompression or stabilization in $4.5 \%$ of cases prior to 2006, and 5.8\%-6.5\% after 2006. This increase in the likelihood of recommending surgery likely occurred as a result of improvements in surgical technique and technological advancements that allowed for a more favorable complication profile with surgery.

The results of this study lay the foundation for further study regarding risk factors and impact of SREs in NSCLC patients. Currently, there is interest in genetic markers and other biomarkers to determine prognosis and predict out- comes in the field of cancer research. These biomarkers can also be utilized as potential therapeutic targets to decrease mortality or to prevent morbidity. Given the current population's similarity to other studied populations of NSCLC patients, the future direction of research at this institution will be to study the genotypes of the tumors in the population to determine if there is commonality in the tumor types that cause significant skeletal and/or spine-related morbidity.

The current study confirms the prevalence and impact of SREs in patients with NSCLC. The spine surgeon is commonly involved in the care of these challenging patients given the predilection of bone metastases to the spine; however, despite this fact there is lack of published data on spine-specific skeletal morbidity and mortality in this population. The present study serves as the basis for future studies on prognostic factors and biomarkers that can influence the survival and quality of life of lung cancer patients.

\section{Conclusions}

SREs in NSCLC patients with bone metastasis are associated with an increased incidence rate of mortality.

\section{Key Points}

Patients with NSCLC often present with spinal metastases. SREs continue to pose a significant challenge despite improvements in the systemic treatment of NSCLC with the refinement of chemotherapeutic protocols. In this single-institution retrospective study, SREs in NSCLC patients with bone metastasis predicted a poor prognosis.

\section{References}

1. Bray F, Ferlay J, Soerjomataram I, et al. Global cancer statistics 2018: GLOBOCAN estimates of incidence and mortality worldwide for 36 cancers in 185 countries. CA Cancer J Clin. 2018;68(6):394-424. 
2. Coleman RE. Skeletal complications of malignancy. Cancer 1997;80(8)(suppl):1588-1594.

3. Cetin K, Christiansen CF, Jacobsen JB, et al. Bone metastasis, skeletal-related events, and mortality in lung cancer patients: a Danish population-based cohort study. Lung Cancer. 2014;86(2):247-254.

4. Delea TE, McKiernan J, Brandman J, et al. Impact of skeletal complications on total medical care costs among patients with bone metastases of lung cancer. J Thorac Oncol. 2006; 1(6):571-576.

5. Sun JM, Ahn JS, Lee S, et al. Predictors of skeletal-related events in non-small cell lung cancer patients with bone metastases. Lung Cancer. 2011;71(1):89-93.

6. Sathiakumar N, Delzell E, Morrisey MA, et al. Mortality following bone metastasis and skeletal-related events among patients 65 years and above with lung cancer: a populationbased analysis of U.S. Medicare beneficiaries, 1999-2006. Lung India. 2013;30(1):20-26.

7. Delea T, Langer C, McKiernan J, et al. The cost of treatment of skeletal-related events in patients with bone metastases from lung cancer. Oncology. 2004;67(5-6):390-396.

8. Pockett RD, Castellano D, McEwan P, et al. The hospital burden of disease associated with bone metastases and skeletalrelated events in patients with breast cancer, lung cancer, or prostate cancer in Spain. Eur J Cancer Care (Engl). 2010; 19(6):755-760.

9. Tsuya A, Kurata T, Tamura K, Fukuoka M. Skeletal metastases in non-small cell lung cancer: a retrospective study. Lung Cancer. 2007:57(2):229-232.

10. Sekine I, Nokihara H, Yamamoto N, et al. Risk factors for skeletal-related events in patients with non-small cell lung cancer treated by chemotherapy. Lung Cancer. 2009;65(2): $219-222$
11. da Silva GT, Bergmann A, Thuler LCS. Incidence and risk factors for bone metastasis in non-small cell lung cancer. Asian Pac J Cancer Prev. 2019;20(1):45-51.

12. Tominaga $\mathrm{H}$, Setoguchi T, Shimada H, et al. Prognostic factors in patients with skeletal-related events at non-small-cell lung cancer diagnosis. Mol Clin Oncol. 2017;7(5):897-902.

\section{Disclosures}

Dr. Buchowski reports receiving royalties from Globus Medical and $\mathrm{K} 2 \mathrm{M} / \mathrm{Stryker}$ and support of a non-study-related clinical or research effort overseen by the author from OMeGA.

\section{Author Contributions}

Conception and design: Adogwa, Buchowski, D’Souza, Shlykov, Jennings. Acquisition of data: Adogwa, D'Souza, Shlykov.

Analysis and interpretation of data: Adogwa, Rubio, Buchowski, D'Souza, Shlykov. Drafting the article: all authors. Critically revising the article: Adogwa, Rubio, Buchowski, Shlykov, Jennings. Reviewed submitted version of manuscript: Adogwa, Buchowski, Jennings. Study supervision: Rubio, Jennings.

\section{Correspondence}

Owoicho Adogwa: University of Texas Southwestern Medical School, Dallas, TX. owoicho.adogwa@utsouthwestern.edu. 